\title{
A Dual Channel X-ray Spectrometer for Fast Ignition Research
}

K. U. Akli, P. K. Patel, R. Van Maren, R. B. Stephens, M. H. Key, D. P. Higginson, B. Westover, C. D. Chen, A. J. Mackinnon, T. Bartal, F. N. Beg, S. Chawla, R. Fedosejevs, R. R. Freeman, D. S. Hey, G. E. Kemp, S. LePape, A. Link, T. Ma, A. G. MacPhee, H. S. McLean, Y. Ping, Y. Y. Tsui, L. D. Van Woerkom, M. S. Wei, T. Yabuuchi

April 21, 2010

Journal of Instrumentation 
This document was prepared as an account of work sponsored by an agency of the United States government. Neither the United States government nor Lawrence Livermore National Security, LLC, nor any of their employees makes any warranty, expressed or implied, or assumes any legal liability or responsibility for the accuracy, completeness, or usefulness of any information, apparatus, product, or process disclosed, or represents that its use would not infringe privately owned rights. Reference herein to any specific commercial product, process, or service by trade name, trademark, manufacturer, or otherwise does not necessarily constitute or imply its endorsement, recommendation, or favoring by the United States government or Lawrence Livermore National Security, LLC. The views and opinions of authors expressed herein do not necessarily state or reflect those of the United States government or Lawrence Livermore National Security, LLC, and shall not be used for advertising or product endorsement purposes. 


\title{
A Dual Channel X-ray Spectrometer for Fast Ignition Research
}

\author{
K. U. Akli ${ }^{1 *}$, P. K. Patel ${ }^{2}$, R. Van Maren ${ }^{2}$, R. B. Stephens ${ }^{1}$, M. H. Key ${ }^{2}$, D. P. \\ Higginson $^{3}$, B. Westover ${ }^{3}$, C. D. Chen ${ }^{2}$, A. J. Mackinnon ${ }^{2}$, T. Bartal ${ }^{3}$, F. N. Beg ${ }^{3}$, S. \\ Chawla $^{3}$, R. Fedosejevs ${ }^{4}$, R. R. Freeman ${ }^{5}$, D. S. Hey ${ }^{2}$, G. E. Kemp ${ }^{5}$, S. LePape ${ }^{2}$, A. \\ Link $^{5}$, T. Ma ${ }^{2}$, A. G. MacPhee ${ }^{2}$, H. S. McLean ${ }^{2}$, Y. Ping ${ }^{2}$, Y. Y. Tsui ${ }^{4}$, L. D. Van \\ Woerkom $^{5}$, M. S. Wei ${ }^{3}$, T. Yabuuchi ${ }^{3}$ \\ ${ }^{1}$ General Atomics, San Diego, CA, 92186, USA, \\ ${ }^{2}$ Lawrence Livermore National Laboratory, Livermore, CA 94550, USA, \\ ${ }^{3}$ University of California San Diego, San Diego, CA, 92186, USA, \\ ${ }^{4}$ University of Alberta, Edmonton, Alberta, Canada T6G 2V4, \\ ${ }^{5}$ Ohio State University, Columbus Ohio, 43210, USA, \\ E-mail: aklilellnl.gov
}

\begin{abstract}
A new Dual Channel Highly Ordered Pyrolytic Graphite (DC-HOPG) x-ray spectrometer was developed to study laser-generated electron beam transport. The instrument uses a pair of graphite crystals and has the advantage of simultaneously detecting self emission from low- $Z$ materials in first diffraction order and high- $Z$ materials in second order. The emissions from the target are detected using a pair of parallel imaging plates positioned in a such way that the noise from background is minimized and the mosaic focusing is achieved. Initial tests of the diagnostic on Titan laser $\left(I \sim 10^{20} \mathrm{~W} / \mathrm{cm}^{2}, \tau=0.7 \mathrm{ps}\right)$ show excellent signal-to-noise ratio (SNR) $>1000$ for the low energy channel and SNR $>400$ for the high energy channel.
\end{abstract}

KEYWORDS: Plasma diagnostics - interferometry, spectroscopy and imaging.

${ }^{*}$ Corresponding author. 


\section{Contents}

1. Introduction 1

2. Dual Channel HOPG Spectrometer (DC-HOPG) Description 2

3. Experimental setup 4

4. Qualification and Performance of the DC-HOPG 4

4.1 Initial tests of the DC-HOPG on the Titan laser 4

4.1.1 The low energy channel 4

$\begin{array}{ll}\text { 4.1.2 The high energy channel } & 6\end{array}$

4.2 Resolving power of the DC-HOPG 7

4.3 Detector orientation and Mosaic focusing 8

$\begin{array}{lll}4.4 & \text { Signal-to-noise ratio (SNR) } & 8\end{array}$

5. Absolute Calibration $\quad 10$

6. Conclusion 12

\section{Introduction}

The interaction of a short pulse high intensity laser with solid targets leads to the generation of energetic electrons. The transport of these fast electrons and their isochoric heating of matter is of great interest to fast ignition $[1,2]$. The flux of laser generated electrons is usually determined from the induced K-shell emission using doped targets [3] or tracer layer techniques [4]. When the electron beam propagates through the target, $\mathrm{K}$-shell emission characteristic of dopant or tracer layer element is produced via electron impact ionization. The coupling efficiency of laser energy to these fast electrons and the energy deposited in the fuel can be inferred from transport models using the experimentally measured $K_{\alpha}$ yields $[3,5,6]$.

The most widely used diagnostic for the absolute K-shell emission is a single hit CCD spectrometer [7]. However this diagnostic has a low dynamic range, does not always operate in the single-photon counting regime, and is susceptible to background and noise associated with high energy, high intensity experiments. Spherically bent Bragg imagers can also collect $K_{\alpha}$ emission and the absolute yield can be determined if the crystal reflectivity is known. The sensitivity of the collection efficiency to target temperature complicates the analysis and limits this technique to cold target where the temperature effects are negligible [8].

Mosaic graphite crystals have high reflectivity compared to other crystals and they have been used to collect weak signals in X-ray Thomson scattering experiments [9] and have also been 
used to record $\mathrm{K}$ shell spectra in fast ignition experiments [5] . In fast ignition experiments, the interaction of kilojoule lasers with the target generates copious amount of noise and background radiation which can mask the desired signal. Shielding of the instrument is therefore important .

In this paper, we report on a newly developed dual channel x-ray spectrometer based on highly ordered pyrolytic graphite crystals. Our novel design uses an optimal configuration of crystals, detectors, and shielding to enhance the signal-to-noise ratio (SNR). This instrument has a high dynamic range and is capable of simultaneously detecting $\mathrm{K}$-shell emission from two different tracer layers. The new design has the advantage of achieving mosaic focusing and excellent shielding of detectors from background radiation. The use of graphite crystals as dispersive optics and imaging plates as detectors gives high dynamic range. It eliminates the need to be in single photon counting regime, the plasma temperature effects, and failure of acquisition by CCD's associated with electromagnetic pulses (EMP).

\section{Dual Channel HOPG Spectrometer (DC-HOPG) Description}

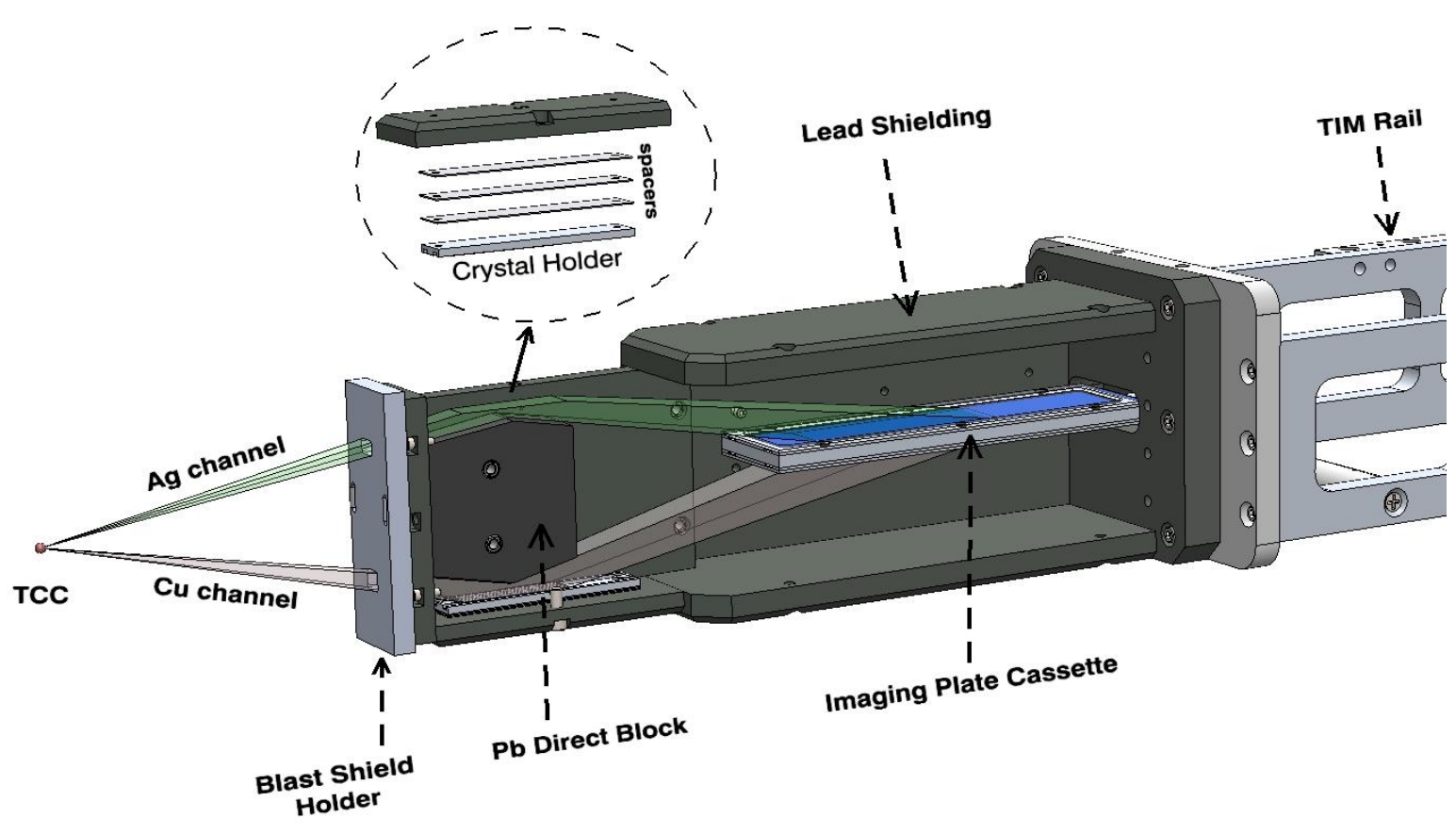

Figure 1. Dual Channel Highly Ordered Pyrolytic Graphite (DC-HOPG) layout

The Dual Channel HOPG Spectrometer (DC-HOPG) consists of two entrance slits, two crystals, a detector cassette, a removable blast shield, a direct light block, a $\mathrm{Pb}$ shielding enclosure, and alignment pin (Fig. 1). The design allows for two x-ray energy channels. A low energy " $\mathrm{Cu}$ channel" optimized for $\mathrm{Cu} K_{\alpha}$ in first diffraction order and a high energy "Ag channel"optimized for $\mathrm{Ag} K_{\alpha}$ in second diffraction order. X-rays emanating from the source enter the spectrometer via two entrance slits. The slits dimensions are chosen such that photon collection efficiency is maximized. The fan of rays entering the spectrometers via the slits fill the entire crystals thereby increasing the amount of photons collected in the non dispersive direction. The slits ensure that no 
marginal rays are seen by the internal components of the instrument other than the crystals thereby minimizing background noise.

X-rays that satisfy the Bragg condition, $n \lambda=2 d \sin (\theta)$, are then diffracted with two highly ordered pyrolytic graphite crystals. The crystals are high purity graphite monochromators, ZYA grade with 0.4 degrees mosaic spread, purchased from Momentive Performance Materials Quartz, Inc. These crystals were mounted on adjustable, removable plates to allow for the use of various crystals and fine tuning of the instrument. The upper bound on the dimensions of the crystals that can be used are 3 inches in length and 0.5 inch in width. With 3 inch long crystals, the Bragg angles of $\mathrm{x}$-rays diffracting from the center of the monochromators are 11.9 degrees for the $\mathrm{Cu}$ channel and 9.7 degrees for the Ag channel.

Table 1. List of some of the characteristic emission lines within the spectral range of the DC-HOPG

\begin{tabular}{cc}
\hline \hline "Cu" Channel: E (eV) & "Ag" Channel: E (eV) \\
\hline $\mathrm{Ni} K_{\alpha 1,2} \rightarrow 7461,7478$ & $\mathrm{Ru} K_{\alpha 1,2} \rightarrow 19150,19279$ \\
$\mathrm{Cu} K_{\alpha 1,2} \rightarrow 8028,8048$ & $\mathrm{Pd} K_{\alpha 1,2} \rightarrow 21020,21177$ \\
$\mathrm{Zn} K_{\alpha 1,2} \rightarrow 8616,8639$ & $\mathrm{Ag} K_{\alpha 1,2} \rightarrow 21990,22163$ \\
$\mathrm{Ga} K_{\alpha 1,2} \rightarrow 9225,9252$ & $\mathrm{Cd} K_{\alpha 1,2} \rightarrow 22984,23174$ \\
\hline $\mathrm{Ni} K_{\beta 1} \rightarrow 8265$ & $\mathrm{Ru} K_{\beta 1} \rightarrow 21657$ \\
$\mathrm{Cu} K_{\beta 1} \rightarrow 8905$ & $\mathrm{Pd} K_{\beta 1} \rightarrow 23819$ \\
$\mathrm{Zn} K_{\beta 1} \rightarrow 9572$ & $\mathrm{Ag} K_{\beta 1} \rightarrow 24942$ \\
$\mathrm{Ga} K_{\beta 1} \rightarrow 10264$ & $\mathrm{Cd} K_{\beta 1} \rightarrow 26096$ \\
\hline Ni Heliumlike $\rightarrow 7766,8102$ & $\mathrm{Ru}$ Heliumlike $\rightarrow 19717,19904$ \\
$\mathrm{Cu}$ Heliumlike $\rightarrow 8347,8392$ & $\mathrm{Pd}$ Heliumlike $\rightarrow 21622,21843$ \\
$\mathrm{Zn}$ Heliumlike $\rightarrow 8950,8999$ & $\mathrm{Ag}$ Heliumlike $\rightarrow 22609,22851$ \\
$\mathrm{Ga}$ Heliumlike $\rightarrow 9575,9628$ & $\mathrm{Cd}$ Heliumlike $\rightarrow 23621,23884$ \\
\hline \hline
\end{tabular}

Listed in table. 1 are some of the characteristic emission lines that can be detected, individually or simultaneously, with this instrument. The maximum achievable spectral range is,

$$
7500 \mathrm{eV} \leq \frac{E}{n} \leq 10500 \mathrm{eV}, \quad n \geq 1
$$

for the low energy channel and,

$$
9500 \mathrm{eV} \leq \frac{E}{n} \leq 13300 \mathrm{eV}, \quad n \geq 1
$$

for the high energy channel, where $\mathrm{n}$ is the diffraction order.

The $\mathrm{x}$-ray spectra are recorded on two parallel imaging plates. The two imaging plates are separated by $5.3 \mathrm{~mm}$ thick $\mathrm{Al}$ plate to prevent the penetration of $\mathrm{x}$-rays from one detector to the other. This thickness is more than sufficient to stop the high energy $22 \mathrm{keV} \mathrm{Ag} K_{\alpha}$ which has 1.6 $\mathrm{mm}$ attenuation length in Al. The imaging plates are placed in a light tight cassette that can be easily removed and interchanged with a new one for the next shot. Both the imaging plate cassette and the slits are covered with $50 \mu \mathrm{m}$ aluminized Mylar to ensure that no visible light reaches the detectors. The aluminized Mylar also protects the crystals from debris damage. A $7 \mathrm{~cm}$ long lead 
block is placed between the source and imaging plates to prevents the direct beam from the source from reaching the detector. This configuration minimizes the exposure of detectors to hard $\mathrm{x}$-rays and $\gamma$ rays. The instrument is housed in $\frac{1}{2}^{\prime \prime}$ thick $\mathrm{Pb}$ body to protect it from background radiation generated during high power laser interaction with the targets.

\section{Experimental setup}

The qualification and flux calibration of the DC-HOPG were carried out on the Titan laser at Lawrence Livermore National Laboratory. Various flat targets were irradiated with either 150J delivered in $0.7 \mathrm{ps}$ or $300 \mathrm{~J}$ delivered in $20 \mathrm{ps}$ pulses. The laser beam irradiated the flat foils at angle of 16 degrees with respect to the normal (Fig. 2). The beam was focused with an $\mathrm{f} / 3$ offaxis parabola with $50 \%$ of the energy contained in $15 \mu \mathrm{m}$ fwhm spot. The spontaneous stimulated emission (ASE) contrast ratio was $10^{-8}$ in intensity and $10^{-4}$ in energy.

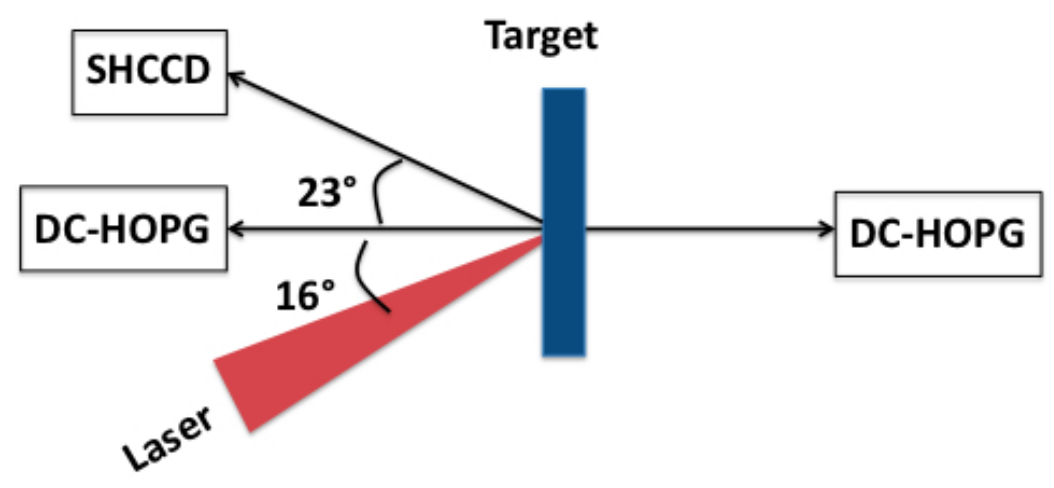

Figure 2. Experimental set up

Three instruments (Two DC-HOPGs and a single hit spectrometer) were fielded to detect x-ray emission from the target. The two DC-HOPGs were positioned along the normal to the target: one looking at the front (irradiated) side, the other at the rear side (Fig. 2). The distance from the target chamber center to the center of each crystal was $21 \mathrm{~cm}$. The single hit CCD spectrometer had a viewing angle of 23 degrees with respect to target normal.

\section{Qualification and Performance of the DC-HOPG}

\subsection{Initial tests of the DC-HOPG on the Titan laser}

\subsubsection{The low energy channel}

The Initial tests of the low energy channel of the DC-HOPG were carried out with $25 \mu m$ thick $\mathrm{Cu}$ targets. Fig. 3-a shows a typical spectrum from a $\mathrm{Cu}$ foil. Fig. 3-b and Fig. 3-c show the front and rear DC-HOPG lineouts. Clearly seen are the $K_{\alpha}$ and $K_{\beta}$ emission lines corresponding to $1 s \rightarrow 2 p_{1 / 2}$ and $1 s \rightarrow 2 p_{3 / 2}$ transitions. In between we see the $H e_{\alpha}$ and $L y_{\alpha}$ thermal lines emitted by highly ionized $\mathrm{Cu}$. The difference in thermal line intensities of rear and front spectra are due to opacity effects [10]. Cu targets oriented in such way that both instruments are looking at the 
same side of the target showed that both DC-HOPGs recorded similar signals to within 5\%. This indicates that the crystals used are of the same quality and have similar reflectivities. A lineout of $K_{\alpha}$ in the non-dispersive direction shows a fairly uniform profile with an average value of 44 and standard deviation of 4 . This result will be used in the calibration process to simplify the integrated $K_{\alpha}$ yield calculations.

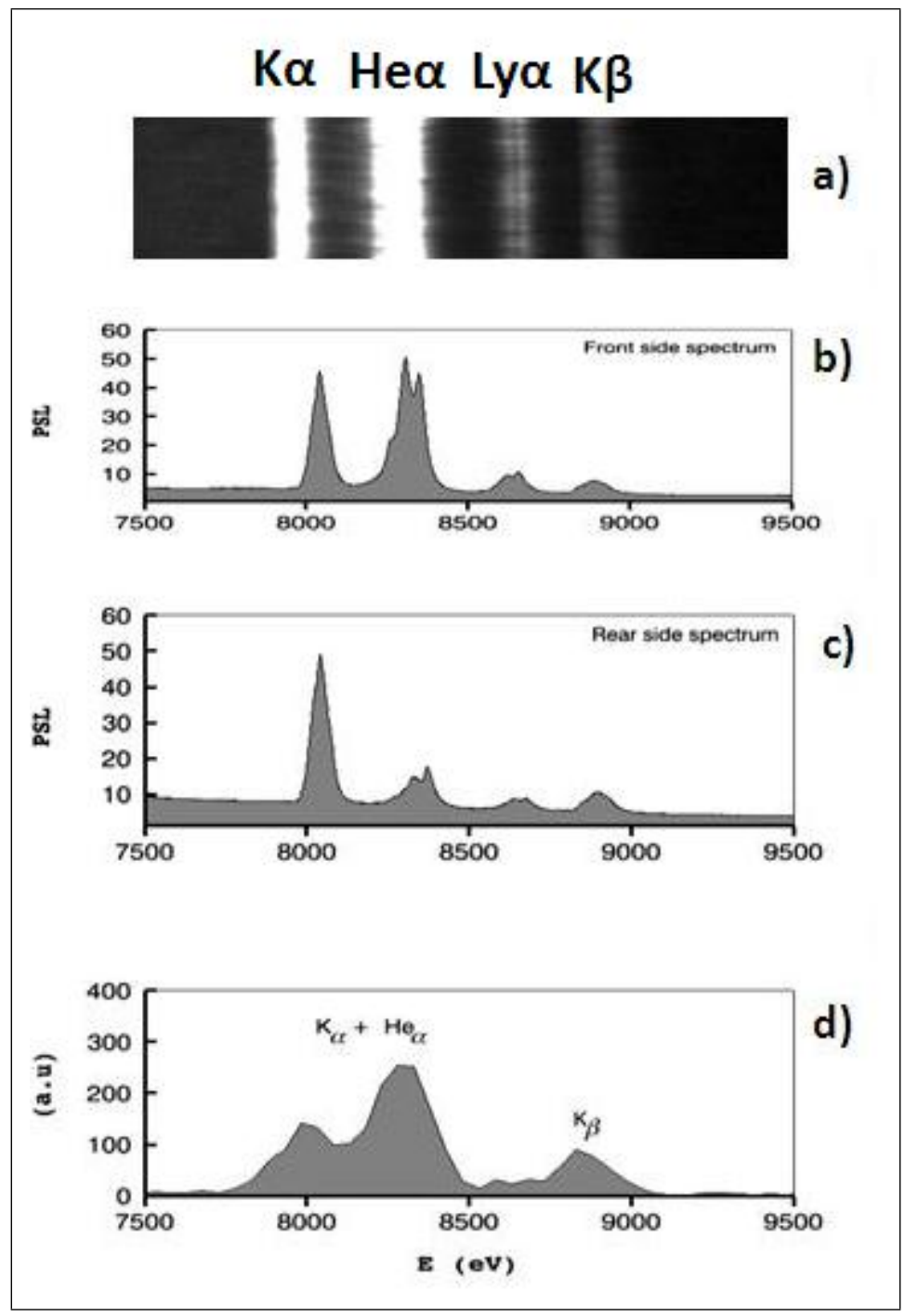

Figure 3. a) Typical spectrum from a $25 \mu \mathrm{m}$ thick $\mathrm{Cu}$ target irradiated by $150 \mathrm{~J} 0.7 \mathrm{ps}$ laser beam, b) Front side DC-HOPG lineout, c) Rear side DC-HOPG lineout, d) A single hit CCD image histogram of similar target under similar irradiation conditions viewed from the front side.

We have compared the performance of the DC-HOPGs to that of the charged coupled devices (CCD) under the same experimental conditions. In addition to their linear response and dynamic range, CCDs have the advantage of electronic data acquisition. This is very convenient for immediate viewing and processing of data. However, our data show that CCDs are neither as reliable nor as useful as DC-HOPGs in acquiring data in high power laser experiments. 
First, the single photon counting condition is only fulfilled for a limited dynamic range . In principle, this can be rectified for changing signal levels by changing the filtering and/or increasing the distance from target to detector. This is inconvenient in single shot experiments on large scale facility where every shot counts. Moreover, the single photon counting condition is highly dependent on both target type and irradiation conditions and there is no guarantee that if it is satisfied for one shot it will be satisfied for the next.

Second, due to its poor spectral resolution, the CCD cannot resolve the $K_{\alpha}$ and $\mathrm{He}_{\alpha}$ emission lines. A comparison of the DC-HOPG spectrum (Fig. 3-b) and CCD image histogram (Fig. 3c) clearly shows the advantage of the former over the later. A CCD histogram will overestimate $K_{\alpha}$ photon flux due to $H e_{\alpha}$ contribution. The DC-HOPG spectrometer not only has the ability to resolve $K_{\alpha}$ and $H e_{\alpha}$, but also it can detect a wide range of emission lines. Intensity ratios, shifting, and broadening of these lines can be used to get valuable information about temperature and density of the plasma $[8,11]$.

Finally, high noise and failure of acquisition have been observed in our experiments for the CCDs, in contrast, the DC-HOPGs have been acquiring data reliably on every shot.

\subsubsection{The high energy channel}

In the case of Ag targets, only $K_{\alpha}$ and $K_{\beta}$ emission line are recorded with both spectrometers ( Fig. 4). The absence of thermal lines indicates that the high $\mathrm{Z}$ silver targets did not get hot enough for these lines to be emitted. The recorded $\mathrm{Ag}$ emission lines show much broader profiles compared to the $\mathrm{Cu}$ lines. Moreover, the $\mathrm{Ag}$ profiles exhibited an asymmetric behavior with features on the high energy side of the emission lines. This volumetric broadening is caused by the significant penetration depth of the photons [12].

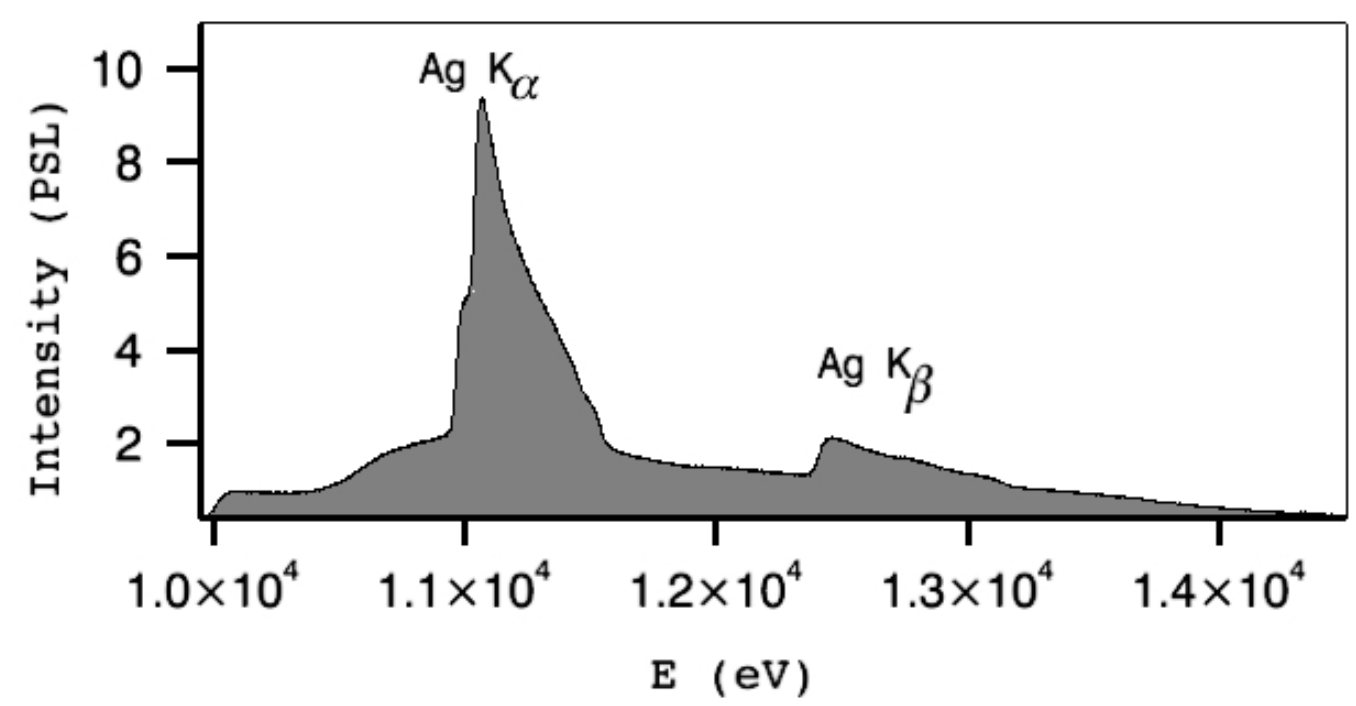

Figure 4. Typical Ag spectrum from a $12 \mu \mathrm{m}$ thick Ag target irradiated by $150 \mathrm{~J} 0.7 \mathrm{ps}$ laser beam 


\subsection{Resolving power of the DC-HOPG}

The factors affecting the resolution can be broadly classified as broadening due to the source, crystal, and detector. The observed spectral line is a convolution of the source broadened profile and the instrument function. The measured resolving power $\Delta E / E$ of the DC-HOPG is $7.46 \cdot 10^{-3}$ for $\mathrm{Cu} K_{\alpha}$ and $1.22 \cdot 10^{-2}$ for $\mathrm{Ag} K_{\alpha}$. It can be expressed by the following quadrature sum,

$$
\left[\frac{\Delta E}{E}\right]^{2}=\left[\frac{\Delta E}{E}\right]_{s r}^{2}+\left[\frac{\Delta E}{E}\right]_{n a t}^{2}+\left[\frac{\Delta E}{E}\right]_{p l}^{2}+\left[\frac{\Delta E}{E}\right]_{m o s}^{2}+\left[\frac{\Delta E}{E}\right]_{v o l}^{2}+\left[\frac{\Delta E}{E}\right]_{r g h}^{2}+\left[\frac{\Delta E}{E}\right]_{d e t}^{2}
$$

The first term in eq (1) refers to the effective source size effect. For a source size of $80 \mu \mathrm{m}$, determined from 2D spatially resolved $K_{\alpha}$ imaging, the source broadening is $20 \mathrm{eV}$ and $42 \mathrm{eV}$ for $\mathrm{Cu} K_{\alpha}$ and $\mathrm{Ag} K_{\alpha}$ respectively. The second term refers to the natural spectral width which is about $2 \mathrm{eV}$ for $\mathrm{Cu} K_{\alpha}$ lines [13] and $10 \mathrm{eV}$ for $\mathrm{Ag} K_{\alpha}$ [14] . The plasma source spectral distribution is complicated and depends on the local plasma characteristics. Several mechanisms contribute to the broadening and shifting of the emission lines. These include doppler, pressure, self-absorption, and ionization broadenings [11]. For our specific case of a $25 \mu \mathrm{m}$ thick $\mathrm{Cu}$ foil irradiated by 150 $\mathrm{J}$ laser, doppler broadening is about $2.5 \mathrm{eV}$ at a typical temperature of $300 \mathrm{eV}$. The radiative and plasma effects are small compared to the extended source effects and are neglected in subsequent analysis. The fourth, fifth, and sixth terms in eq (1) are the broadenings due to the mosaicity, depth, and surface roughness of the graphite crystal. Their effects on the resolution have been extensively discussed by $[15,16]$. Ray tracing results specific to our spectrometer are summarized in the table below.

Table 2. Ray Tracing Results

\begin{tabular}{ccccc}
\hline \hline Broadening & $\mathrm{Cu} K_{\alpha}(\Delta E \mathrm{eV})$ & $\Delta E / E$ & $\mathrm{Ag} K_{\alpha}(\Delta E \mathrm{eV})$ & $\Delta E / E$ \\
\hline Source size & 25 & $2.485 \cdot 10^{-3}$ & 42 & $1.668 \cdot 10^{-3}$ \\
Mosaic & 7.1 & $8.722 \cdot 10^{-4}$ & 36.9 & $1.098 \cdot 10^{-3}$ \\
Depth & 45 & $5.881 \cdot 10^{-3}$ & 243.4 & $1.373 \cdot 10^{-3}$ \\
Roughness & 7.9 & $9.814 \cdot 10^{-4}$ & 30.4 & $6.963 \cdot 10^{-4}$ \\
Detector & 4.1 & $5.036 \cdot 10^{-3}$ & 15.4 & $1.895 \cdot 10^{-3}$ \\
\hline Total & 52.7 & $6.537 \cdot 10^{-3}$ & 252.1 & $1.137 \cdot 10^{-2}$ \\
\hline
\end{tabular}

For the low energy channel, the effective source size and crystal depth contribute significantly to the broadening. The width of the $\mathrm{Cu} K_{\alpha}$ line obtained with ray tracing was $\sim 53 \mathrm{eV}$. This is consistent with the experimentally measured width of $56 \mathrm{eV}$. For the high energy channel, the dominant factor affecting the resolution is the crystal thickness. The experimentally measured fwhm of $\mathrm{Ag} K_{\alpha}$ was $270 \mathrm{eV}$ which is also consistent with ray tracing results. Our crystal thickness is $\sim 2 \mathrm{~mm}$ and the resolution could be improved by using a thinner crystal $(100-200 \mu \mathrm{m})$ without significant loss of reflectivity ( $8 \mathrm{keV} \leq E \leq 24 \mathrm{keV}$ ) [17]. For our purpose, where the primary goal is to count $K_{\alpha}$ photons, the obtained resolution is enough. The spectrometer clearly resolves the $K_{\alpha}$ line from the adjacent $\mathrm{He}_{\alpha}$ line. 


\subsection{Detector orientation and Mosaic focusing}

An x-ray beam penetrating the crystal will encounter mosaic blocks such that the Bragg condition is satisfied for a given energy. This property leads to a focusing effect similar to the one in BraggBrentano geometry in powder diffraction [18]). This interesting effect, which is the consequence of crystallites in mosaic crystals, is known as mosaic focusing or parafocusing [15]. Glenzer et al (2003) have demonstrated, in the context of x-ray scattering experiments, that the crystal has to be equidistant from both source and detector in order for the mosaic focusing to be achieved. The orientation of the detector with respect to the diffracted rays does not contribute to the defocusing for single emission lines. However, if multiple lines are of interest as is the case in our experiments, the orientation of the detector is important. Fig. 5-a shows a configuration where the detector is normal to the diffracted rays. It is clear that in this setup, the source-crystal and crystal-detector are equal for one emission line (ray-1) but not for the other (ray-2). The defocusing effects will increase with increasing diffraction order and distances. Our design uses a detector parallel to the crystal (Fig. 5-b). In addition to the advantages that the detector is better shielded from background radiation, this configuration ensures point-to-point parafocusing.

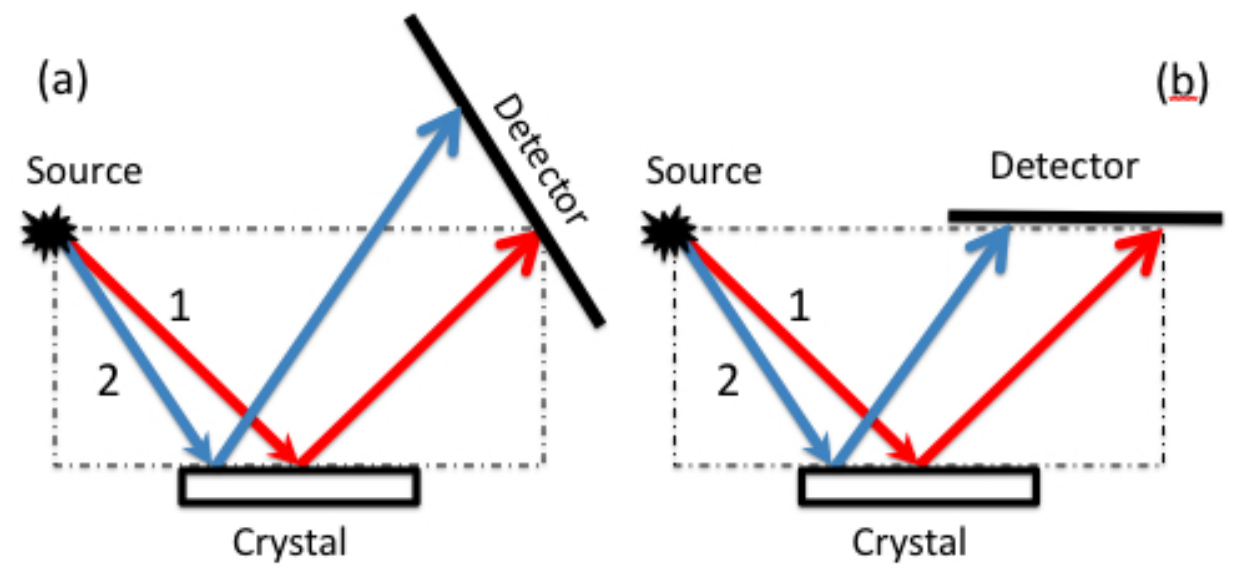

Figure 5. Detector orientation and mosaic focusing: a) Detector normal to the central diffracted ray (Red), b) Detector parallel to the crystal

\subsection{Signal-to-noise ratio (SNR)}

The signal-to-noise ratio was determined by taking the ratio of the background corrected peak signal to signal rms. A single pixel lineout analysis yields an average SNR $\sim 225$ for the low energy channel. However, since the $\mathrm{Cu} K_{\alpha}$ emission line is fairly uniform in the non-dispersive direction, averaging over many pixels conserves the peak signal while the background noise is averaged. This results in an enhanced signal-to-noise ratio. For example, an average lineout using a box with 160 pixels in the non-dispersive direction yields an average SNR $\sim 1000$ (Table. 3). For the high energy channel, the signal-to-noise ratio was lower $\sim 400$ for a box average. This due to the fact that the slit is closer to the equatorial plane of laser-target interaction as the Bragg angle is 
smaller for this channel. Also, the interaction of the laser beam with high $\mathrm{Z}$ targets generates more intense hard x-rays which contribute to background noise.

Table 3. SNR Results

\begin{tabular}{cccccc}
\hline \hline Shot & $\begin{array}{c}\text { Peak } \\
\text { signal }\end{array}$ & $\begin{array}{c}\text { Sing. pix. } \\
\text { Stdev }\end{array}$ & $\begin{array}{c}\text { Sing. pix. } \\
\text { SNR }\end{array}$ & $\begin{array}{c}\text { Box Aver. } \\
\text { Stdev }\end{array}$ & $\begin{array}{c}\text { Box Aver. } \\
\text { SNR }\end{array}$ \\
\hline $20080805-S 1$ & 73.9 & 0.158 & 467 & 0.062 & 1188 \\
$20080805-S 2$ & 36.4 & 0.184 & 198 & 0.024 & 1518 \\
$20080805-S 3$ & 49 & 0.252 & 195 & 0.034 & 1458 \\
$20080806-S 1$ & 40 & 0.276 & 144 & 0.095 & 420 \\
20080806-S2 & 40 & 0.325 & 122 & 0.100 & 400 \\
\hline Average & & 225 & & 1000 \\
\hline
\end{tabular}

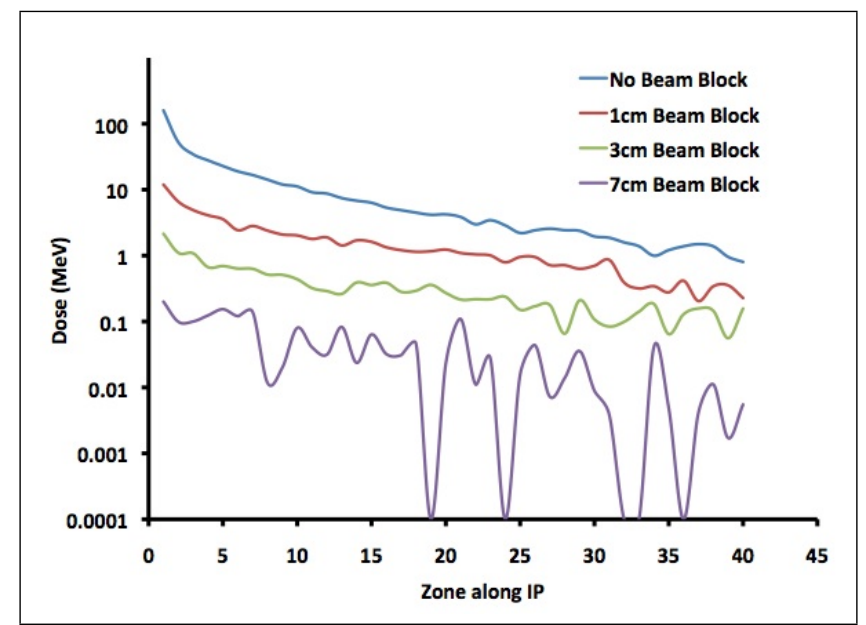

Figure 6. Dose deposited in the imaging plate as function of the zones for three different beam block thicknesses. Each zone is $257 \mu \mathrm{m}$ long

The excellent signal-to-noise ratio of the DC-HOPG is the result of an optimal shielding and detector positioning design. In addition to achieving point-to-point mosaic focusing, the horizontally positioned imaging plates are well shielded by the $7 \mathrm{~cm}$ long $\mathrm{Pb}$ block from direct radiation from the target. We have conducted Monte Carlo simulations of radiation transport through the $\mathrm{Pb}$ beam block using the Monte Carlo code Integrated Tiger Series 3.0 [19]. First, an electron distribution with $1.2 \mathrm{MeV}$ average energy was injected into $250 \mu \mathrm{m}$ thick Ag target. Then, a sample size of 250,000,000 photons were generated in a 15 degree cone directed towards the beam block. The imaging plate has four layers: mylar, phosphor, mylar, and ferrite layer. Each imaging plate was divided into 400 zones and each zone was $350 \mu \mathrm{m}$ wide and $257 \mu \mathrm{m}$ long. After the transport through the $\mathrm{Pb}$ beam block, the simulation recorded the energy deposition in each zone of the phosphor layer. Three different beam block thicknesses were used: $1 \mathrm{~cm}, 3 \mathrm{~cm}$, and $7 \mathrm{~cm}$. Fig. 6 shows the dose on the imaging plate averaged over the width of the detector. The $7 \mathrm{~cm}$ long beam block decreases the dose, due to background radiation, by a factor of 100 compared to the $1 \mathrm{~cm}$ 
long beam block and by a factor of 1000 compared to no beam block. The $\frac{1}{2}^{\prime \prime}$ thick Pb housing of the instrument reduces the background radiation generated from the bulk of target chamber.

\section{Absolute Calibration}

The calibration of the DC-HOPG for photon flux was carried using a Spectral Instruments CCD. This camera was previously calibrated at $5.9 \mathrm{keV}$ and $22 \mathrm{keV}$ using Fe-55 and Cd-109 sources [20]. The SI800 CCD was located at a distance of $5.20 \mathrm{~m}$ at an angle of 23 degrees with respect to the normal to the front surface of the target. For the low energy channel we have used $5 \mu \mathrm{m} \mathrm{Al} / 25 \mu \mathrm{m} \mathrm{Cu}$ targets. The $\mathrm{Cu} K_{\alpha}$ yields from both instruments are listed in Table. 4.

Table 4. Calibration Shots Results

\begin{tabular}{cccc}
\hline \hline Shot & Laser Energy (J) & DC-HOPG $(P S L . e V / J / s r)$ & SHCCD $(p h / J / s r)$ \\
\hline $20090803-S 5$ & 123 & $2.57 \times 10^{6}$ & $6.17 \times 10^{9}$ \\
$20090803-S 6$ & 310 & $3.75 \times 10^{6}$ & $1.36 \times 10^{10}$ \\
\hline
\end{tabular}

For the SHCCD spectrometer, the number of $K_{\alpha}$ photons normalized to laser energy and detector solid angle $(\mathrm{ph} / \mathrm{J} / \mathrm{sr})$ is determined by the following expression,

$$
N_{C C D}=\frac{\int h(E) d E}{E_{L} \cdot \varepsilon_{C C D} \cdot T_{\text {filters }} \cdot \Omega_{\text {image }}} \kappa_{o p}
$$

Where $h(E)$ is the background subtracted histogram of the CCD image, $E_{L}$ is the laser energy in Joules, $\varepsilon_{C C D}$ is the product of the quantum efficiency, single hit probability, and crowding correction, $T_{\text {filters }}$ is the transmission of all the filters (including Be windows), $\Omega_{\text {image }}$ is the solid angle of the detector, and $\kappa_{o p}$ is the opacity correction along the line of sight due to reabsorption of the $K_{\alpha}$ emission in the target.

For the DC-HOPG, the number of $K_{\alpha}$ photons normalized to laser energy and detector solid angle (PSL.eV $/ \mathrm{J} / \mathrm{sr}$ ) can be determined according to the following expression,

$$
N_{D C-H O P G}=\frac{\kappa_{o p}}{E_{L} \cdot \Omega_{d e t}} \iint_{D} f(x, y) d x d y
$$

Where $\mathrm{f}(\mathrm{x}, \mathrm{y})$ is the background subtracted $2 \mathrm{D} K_{\alpha}$ emission profile recorded by the detector. D is a region that includes the $K_{\alpha}$ line, its transverse size can be chosen as convenient while its longitudinal extent (in the dispersion direction) is determined by the mosaic spread of the crystal.

In the DC-HOPGs, we used BAS- MS 2040 imaging plates as detectors. After data aqcuisition, the imaging plates were scanned with Fuji FLA7000 scanner. The digitized images were then converted to Photo Stimulated Luminescence units (PSL) using the following formula (Fuji manuel),

$$
P S L=\left[\frac{\text { Res }}{100}\right]^{2} \cdot \frac{4000}{S} \cdot 10^{L\left[\frac{Q L}{G}-\frac{1}{2}\right]}
$$

Our scanning parameters were $50 \mu \mathrm{m}$ for resolution (Res), 4000 for sensitivity (S), 5 for Latitude (L), 65535 for gradation (G), and a range of 0 to 65535 for QL. The PSL converted images are two 
dimensional of the form $f(x, y)$. Since, as mentioned previously, emission lines are fairly uniform in the non-dspersive (x) direction, eq.(5) can be written as,

$$
N_{D C-H O P G}=\frac{\kappa_{o p}}{E_{L} \cdot \Omega_{p i x}} \int f(E) d E
$$

where $\Omega_{p i x}$ is the single pixel lineout solid angle. $\mathrm{f}(\mathrm{E})$ is the average lineout of the $K_{\alpha}$ line obtained after transforming the coordinate space (y) to energy space using the dispersion expression,

$$
\frac{\Delta E}{\Delta y}=\frac{E}{2 F \cdot \tan (\theta)}, F-\text { source to crystal distance }
$$

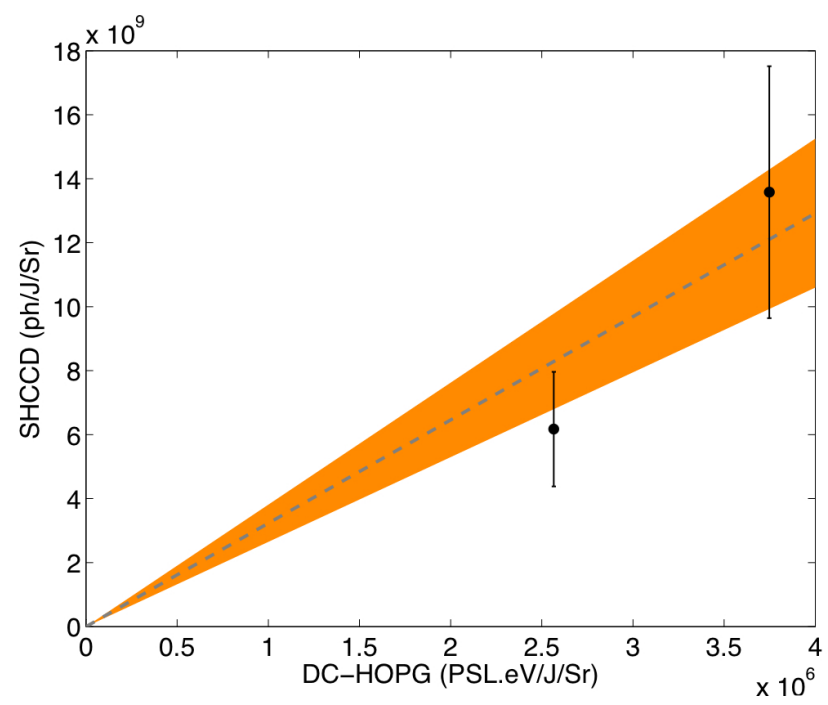

Figure 7. Calibration curve: The dashed line is the calibration curve, the yellow region represents the error in the best fit to the two experimental data points

For a set of data $\left\{X_{i}, Y_{i}\right\}$, where the $X_{i}$ and $Y_{i}$ are the DC-HOPG and SHCCD yields from Table. 4, we seek a weighted least square fit straight line $Y=A X$. The constant $\mathrm{A}$ is the calibration factor which includes the reflectivity of the crystal, filter transmissions, and the response of the imaging plate to $\mathrm{x}$-rays. This constant is determined from the data by minimizing the $\chi^{2}$,

$$
\frac{\partial \chi^{2}}{\partial A}=\frac{\partial}{\partial A} \sum_{i} \frac{1}{\sigma_{i}^{2}}\left\{Y_{i}-A X_{i}\right\}^{2}=0
$$

which gives,

$$
A=3.23 \times 10^{3} \pm 18 \%
$$

The calibration curve is plotted in Fig. 7. The dashed line is the calibration curve $\left(Y=3.23 \times 10^{3} \times\right.$ $X)$. The yellow band represents the error in least square data fit. For the high energy channel calibration, we have used $\mathrm{Al}$ targets with a $25 \mu \mathrm{m} \mathrm{Ag}$ thick layer. Similar analysis yields a calibration constant $A=6.69 \times 10^{3} \pm 50 \%$. 


\section{Conclusion}

In conclusion, we have developed an x-ray spectrometer for fast ignition research. It has low and high energy channels. This instrument is compact and has the advantage of flexibility in the choice of tracer or dopant materials due to the wide spectral range of both channels. The orientation of the imaging plates with respect to the crystals allows point-to-point mosaic focusing to be achieved. The location of the imaging plates and the optimal shielding of the instrument enhances the signalto-noise ratio. The qualification of the DC-HOPG on the Titan laser shows a SNR> 1000 with $\mathrm{Cu}$ foils and SNR $>400$ for Ag targets. The DC-HOPG is absolutely calibrated and its spectral resolution is suitable for the study of laser-generated electrons in the context of fast ignition.

\section{Acknowledgments}

We wish to acknowledge the Titan laser facility staff. Useful discussions with P. Neumayer are acknowledged. This Work was performed under the auspices of the U.S. Department of Energy by the Lawrence Livermore National Laboratory under Contract No. DE-AC52-07NA27344.

\section{References}

[1] Max Tabak et al., Phys. Plasmas 1 (1994), 1626.

[2] M. H. Key, Physics of Plasmas 14 (2007), no. 5, 055502.

[3] M. H. Key et al., Phys. Plasmas 15 (2008), 022701.

[4] R. B. Stephens et al., Phys. Rev. E 69 (2004), 066414.

[5] J. A. King et al., Phys. Plasmas 16 (2009), 020701.

[6] C. D. Chen et al., Phys. Plasmas 16 (2009), 082705.

[7] C. Stoeckl et al., Rev. Sci. Instrum. 75 (2004), 3705.

[8] K. U. Akli et al., Physics of Plasmas 14 (2007), no. 2, 023102.

[9] S. H. Glenzer et al., Physics of Plasmas 10 (2003), no. 6, 2433.

[10] K. U. Akli et al., Phys. Rev. Lett 100 (2008), 165002.

[11] G. Gregori et al., Contrib. Plasma Phys 45 (2005), no. No 3-4, 284-292.

[12] et al T. Doppner, Rev. Sci. Instrum. 79 (2008), 10E311.

[13] J. A. Bearden and C.H. Shaw, Phys. Rev. 48 (1935), 18.

[14] H. E. Welch, X-ray line width measurements with a three-crystal spectrometer, Ph.D. thesis, Texas Technological College, 1969.

[15] G. E. Ice and C. J. Sparks, Nucl. Instrum. Methods Phys. Res. A 291 (1990), 110.

[16] A. Pak et al., Rev. Sci. Instrum. 75 (2004), 3747.

[17] A. K. Freund et al., Proc. SPIE. 68 (1996), 2865.

[18] V. A. Arkadiev et al., Spectrochimica Acta Part B 62 (2007), 577Đ585.

[19] J. A. Halbleib et al., IEEE Trans. Nucl. Sci. 39 (1992), 1025.

[20] B. Maddox et al, Rev. Sci. Instrum. 79 (2008), 10E924. 